\title{
Heated tobacco product
}

Addiction Ontology

\section{Source}

Addiction Ontology

Definition: A tobacco-containing product that contains a tobacco stick that it heats to cause minimal or no combustion to produce smoke or a vapour for inhalation by a person.

Comment: This product is described as not involving combustion of tobacco and thereby avoiding production of harmful chemicals caused by combustion such as carbon monoxide. However for at least some products there is some evidence of a degree of combustion.

Curator note: The entity uses 'has part' rather than 'contains' for the relationship with 'tobacco stick' because the stick is part of the product and not separate from it and included within it.

This definition was imported from the Addiction Ontology (see https://addictovocab.org/). Comments and suggestions for improvements are welcome using the Qeios review system.

Definitions imported from the Addiction Ontology (AddictO) are what are known as 'ontological definitions'. See this article in Qeios for an explanation https://www.qeios.com/read/YGIF9B.

Ontological definitions can sometimes be hard to read. In those cases we also include an informal definition.

Definitions also often require elaboration to make it clear how they should be used and what they include. In those cases we include a comment.

Also, definitions sometimes require an explanation as to how they came about to help users understand how they relate to alternative definitions. In those cases we include a curator note. 